\title{
Dealing With Multiple Grains in TEM Lamellae Thickness for Microstructure Analysis Using Scanning Precession Electron Diffraction
}

\author{
A. Valery ${ }^{1,2}$, E. F. Rauch ${ }^{2}$, A. Pofelski ${ }^{1}$, L. Clément ${ }^{1}$, F. Lorut ${ }^{1}$ \\ 1. STMicroelectronics, 850 rue Jean Monnet, F-38920 Crolles, France. \\ 2. SIMAP/GPM2 laboratory, 101 rue de la Physique, 38402 Saint Martin d'Hères, France.
}

Materials microstructure is a source of variability in devices performance considering today's transistor feature sizes. As the well-known Electron BackScatter Diffraction (EBSD) technique [1] shows limitations to characterize grains orientation smaller than a hundred nanometers, new tools have been developed over the past few years to characterize the texture of crystalline materials at nanometer scale. Among them is the ASTAR tool based on the acquisition and indexation of Precession Electron Diffraction (PED) patterns acquired in scanning mode [2]. Although its use has proved to be efficient to analyze nano-crystalline materials [3], indexation issues appear when crystal grains size is significantly smaller than the lamella thickness. For such cases, the acquired images are composed of a superimposition of several diffraction patterns. Strong expertise and time is required to prepare ultra-thin TEM lamellae adapted for the analysis of current nanometer scale transistor devices so that only the feature of interest remains in the thickness while left crystalline. As an alternative, a dedicated procedure is proposed to overcome crystal overlapping issues and exploit volume information: each diffraction pattern is iteratively re-indexed after subtraction of the reflections related to the main-or previoussolution. In this work, this technique is used to study the microstructure of a material when several crystalline phases remain in the TEM lamella thickness.

The sample observed was a planar section of nickel silicide (NiSi) thin films ( 30nm) on Silicon-OnInsulator (SOI) where some monocrystalline silicon (Si) remained in the thickness of a typical 50-70nm lamella. A scheme of the studied sample is shown in figure 1a. The lamella was prepared using a H450 FEI dual beam system. Experiments were carried out using a FEI Tecnai G2 F20 S-Twin FEG (S)TEM operating at $200 \mathrm{keV}$. A quasi-parallel probe of $4 \mathrm{~nm}$ was used to scan the sample, with a convergence semi-angle measured to be at $0.4 \mathrm{mrad}$. For the acquisition, the sample was oriented along the silicon [001] direction. Scanning electron diffraction and orientation analysis were performed using the ASTAR/DigiSTAR system.

Figures 2a shows an example of a diffraction pattern acquired during the scanning. At least two different diffraction arrays can be observed and retrieved as illustrated in figures $2 b$ and $2 c$. The Si phase comes as the first indexation result and some reflections are clearly recognized as the signature of [001]oriented silicon (figure 2b). After subtraction of the Si-related reflections, the remaining diffraction pattern free of any $\mathrm{Si}$ footprint is available for indexation. Thanks to this re-indexation, a more reliable orientation mapping of NiSi phase [4] can be produced as displayed in figure 1c. In figure 2c, the orientation of a NiSi grain is correctly indexed as a specific simulated template matches the diffraction pattern with a good correlation index. The indexation clearly identifies the morphological aspect of NiSi grains all along the surface. An interesting result is the epitaxial relationship between the two phases as most of the NiSi crystals are oriented along the [001] direction such as $\mathrm{Si}$.

To summarize, a specific method to extract and exploit the multi-information present in diffraction patterns was examined. The experiment demonstrated that this technique allows us to perform the 
relevant characterization of NiSi grains, even if the TEM lamella was not thin enough to include the only phase to observe. This alternative approach can be an efficient answer to avoid the preparation of TEM lamellae as thin as features size and thereby provide keys for microstructure analysis of nanocrystals.

\section{References}

[1] V. Randle, Materials Characterization 60 (2009), p.913-922.

[2] E. F. Rauch, M. Véron, Materials Characterization 98 (2014), p.1-9.

[3] A. Valery et al, International Microscopy Congress Proceedings (2014), p.3205-3206.

[4] M. Kh. Rabadanov and M. B. Ataev, Inorganic Materials 38 (2002), p.120-123.
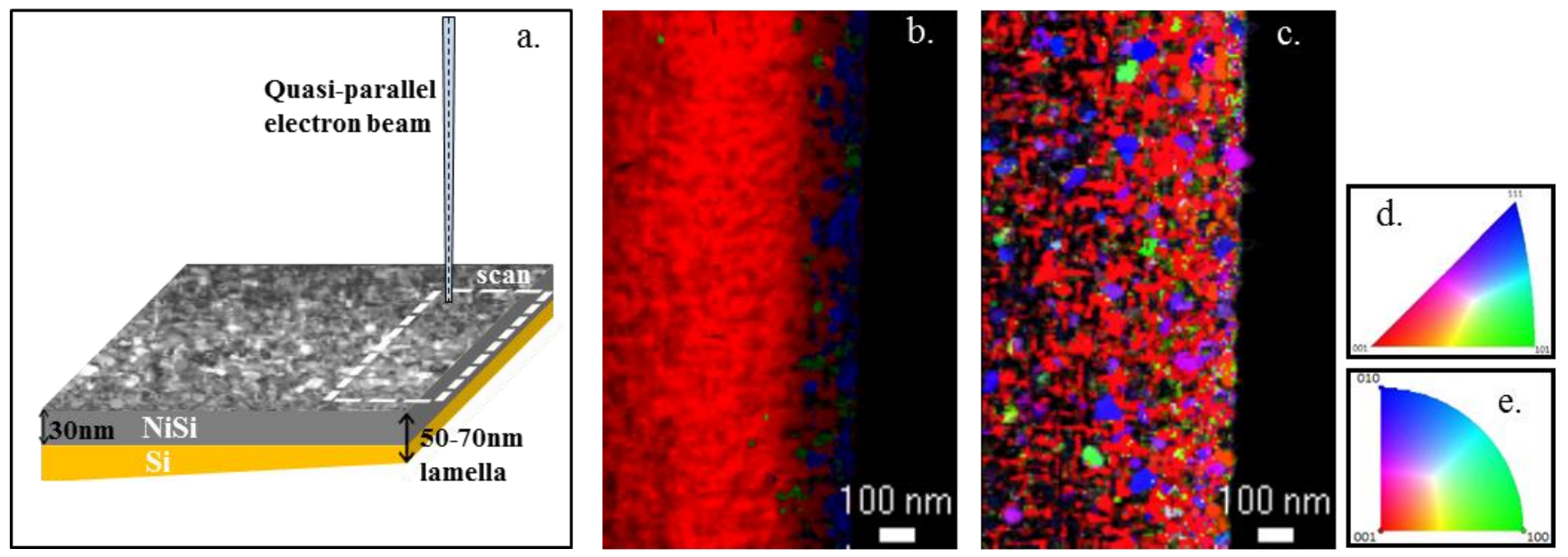

Figure 1. Orientation analysis of the observed TEM lamella, including: (a) a scheme of the prepared planar TEM lamella; (b) the orientation map of the Si remaining in the sample; (c) the orientation map of the NiSi characterized through re-indexation; (d) and (e) the color codes for Si (cubic system) and NiSi (orthorhombic system), respectively.
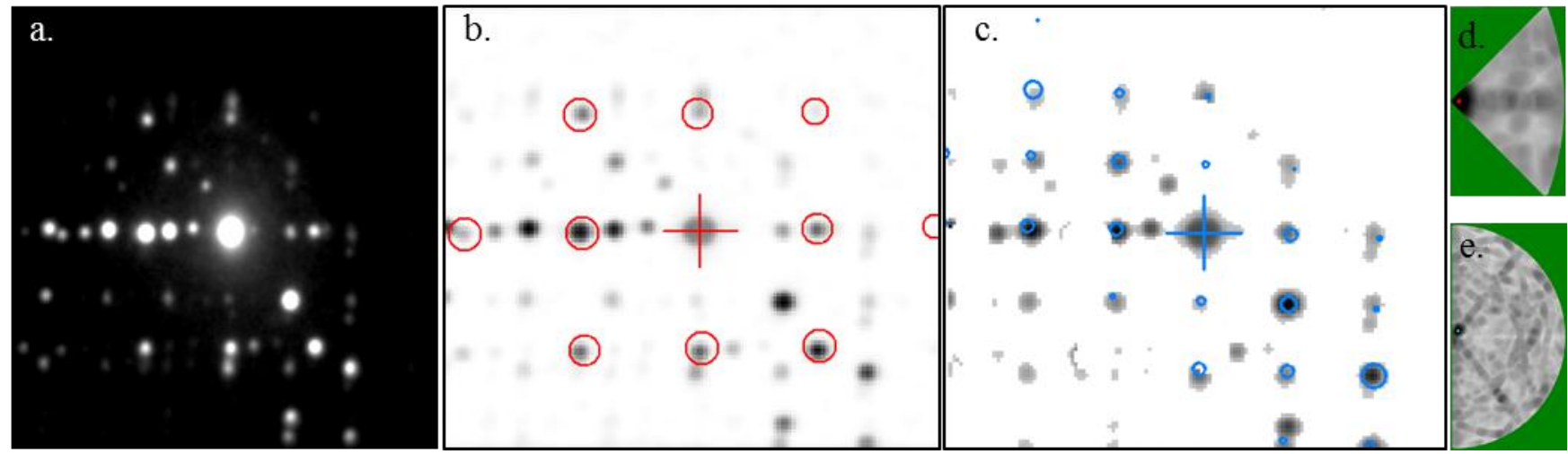

Figure 2. Illustration of a diffraction pattern (re)indexation, with: (a) a diffraction pattern corresponding to a point in the mapping illustrated above; (b) the diffraction spots firstly indexed as Si by template matching; (c) the diffraction spots indexed as NiSi after image treatment; (d) and (e) the ASTAR correlation index maps of the [001]-oriented Si and [001]-oriented NiSi after re-indexation, respectively. 